\title{
Probability Distribution of the Order Parameter
}

\author{
P. H. L. Martins ${ }^{1,2}$ and J. A. Plascak ${ }^{2}$ \\ ${ }^{1}$ Universidade Federal do Tocantins, Caixa Postal 111, 77001-970, Palmas, TO, Brazil \\ ${ }^{2}$ Departamento de Física, Instituto de Ciências Exatas, Universidade Federal de Minas Gerais, \\ Caixa Postal 702, 30123-970, Belo Horizonte, MG, Brazil
}

Received on 1st December, 2003

\begin{abstract}
The probability distribution of the order parameter is analyzed in order to obtain the criticality of magnetic systems. Monte Carlo simulations have been employed by using single spin flip Metropolis algorithm aided by finite-size scaling and histogram reweighting techniques. A method is proposed to obtain this probability distribution even when the transition temperature of the model is unknown. A test is performed on the twodimensional spin-1/2 and spin-1 Ising model and the results show that the present procedure can be quite efficient and accurate to describe the criticality of the system.
\end{abstract}

\section{Introduction}

The order parameter distribution function has been proved to be a powerful tool for studying not only magnetic systems [1-5], but also the liquid-gas critical point [6], the critical point in the unified theory of weak and electromagnetic interactions[7], and the critical point in quantum chromodynamics[8]. For the specific case of magnetic systems the order parameter can be chosen as the magnetization per spin, namely $m=\frac{1}{N} \sum_{i=1}^{N} S_{i}$, where $N$ is the total number of spins and $S_{i}$ is the spin at site $i$. In finitesize systems, the magnetization $m$ is a fluctuating quantity, characterized by the probability distribution $P(m)[1,2]$. In Ising-like models undergoing a second-order phase transition it is known that at temperatures lower than the critical temperature $T_{c}$, the distribution $P(m)$ has a double peak, centered at the spontaneous magnetizations $+m$ and $-m$. At temperatures greater than $T_{c}, P(m)$ has a single peak at zero magnetization, and exactly at $T_{c}$ a double-peak shape is observed[1]. Analogously to the usual finite-size scaling assumptions [9], one then expects that, for a large finite system of linear dimension $L$ at the critical point, $P(m)$ takes the form

$$
P(m)=b P^{*}(\tilde{m})
$$

where $b=b_{0} L^{\beta / \nu}, \beta$ and $\nu$ are critical exponents, $\tilde{m}=b m$, $b_{0}$ is a constant, and $P^{*}(\tilde{m})$ is a universal scaling function, normalized to unit norm and unit variance. Scaling functions, such as $P^{*}$, are characteristic of the corresponding universality class. Systems belonging to the same universality class share the same scaling functions. Thus, from the precise knowledge of $P^{*}(\tilde{m})$ one can characterize critical points and also identify universality classes. This is what has been done so far in the literature, with the distribution for the spin-1/2 Ising model being the standard $P^{*}$ function[4, 6] for this universality class. For instance, it is shown in Fig. 1 the normalized distribution $P^{*}(\tilde{m})$ for the two-dimensional spin-1/2, spin-1, and spin-3/2 Ising model at criticality. Simulations have been done on square lattices with $L=32$ at the exact $T_{c}$ for spin-1/2, at $T_{c}=1.6935$ for spin- 1 , according series expansions [10] and Monte Carlo simulations [11], and at $T_{c}=3.28794$ for the spin-3/2 model [11]. The universal aspect of these systems can be easily noted.

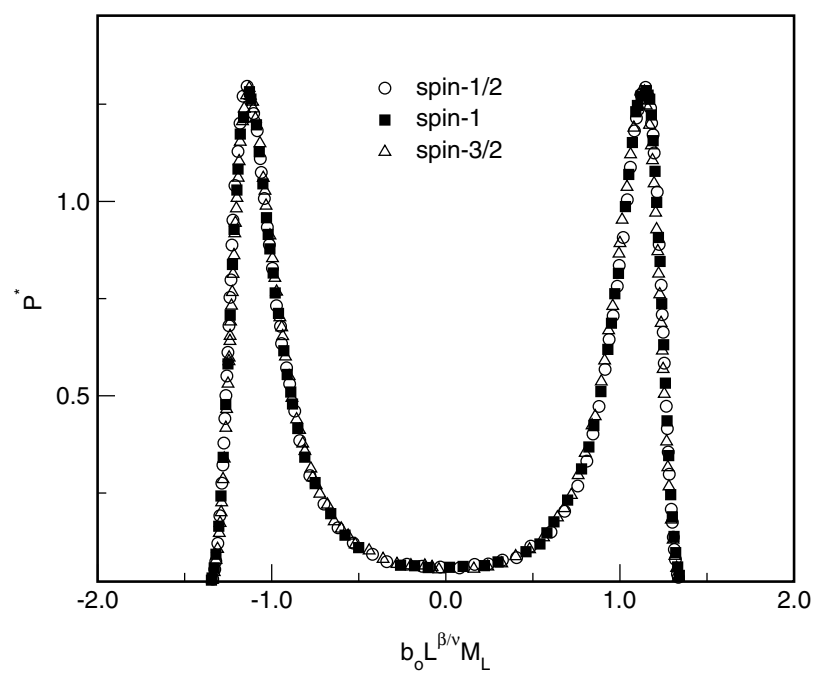

Figure 1. Scaling function $P^{*}(\tilde{m})$ for the two-dimensional spin$1 / 2$, spin- 1 , and spin-3/2 Ising model on square lattices with $L=$ 32. Simulations were performed at the exact $T_{c}$ for spin- $1 / 2$, at $T_{c}=1.6935$ for spin-1, according series expansions [10] and Monte Carlo simulations [11], and at $T_{c}=3.28794$ for the spin$3 / 2$ model [11]. The error bars are smaller than the symbol sizes. After Ref. [11]

Monte Carlo simulations seem to be the most effective method to obtain results as those shown in Fig. 1, where the probability distribution $P(m)$ corresponds to the fraction of the total number of realizations in which the system magne- 
tization is $m$, i.e.,

$$
P(m)=\frac{N_{m}}{\mathcal{N}_{M C S}}
$$

where $N_{m}$ is the number of times that magnetization $m$ appears and $\mathcal{N}_{M C S}$ is the total number of Monte Carlo steps. To compute the normalized distribution $P^{*}(\tilde{m})$ via Eq. (1) one has to evaluate the pre-factor $b$. This can be easily done by noting that $b=1 / \sigma$, where $\sigma$ is the square root of the magnetization variance $\left(\sigma^{2}=\left\langle m^{2}\right\rangle-\langle m\rangle^{2}\right)$. Thus, one obtains the universal function $P^{*}(\tilde{m})$ by simply rescaling the magnetization and by using Eq. (1).

In general, the probability distribution is used for studying models in which the critical temperature or even the distribution function is exactly (or high-precisely) known. That is in fact what has been done in the study of several systems. When this distribution, as well as the critical temperature and critical exponents, are not known, one can of course do first a canonical simulation in order to get the critical values (universal and non universal) and compute, afterwards, the desired distribution. The present approach is different from this conventional one in the sense that it does use the order parameter distribution itself in order to obtain the criticality of the system. The procedure, as well as the results obtained for the spin-1/2 and spin-1 Ising model, are discussed in section 2 and the conclusions are presented in the final section.

\section{Approach and results}

We have performed extensive Monte Carlo simulations (up to $10^{7}-10^{8}$ Monte Carlo steps per spin after $2.0-5.0 \times 10^{4}$ steps for thermalization) on square $L \times L$ lattices with periodic boundary conditions for systems of length $12 \leq L \leq$ 64 . For a given $L$, the simulation ran at a fixed temperature, evolving according the standard Metropolis algorithm. A histogram reweighting technique $[12,13]$ was used to obtain thermodynamic information in the vicinity of the simulated temperature.

Let us first discuss the spin-1/2 Ising model. Fig. 2 shows the distribution $P^{*}$ as a function of the normalized magnetization $\tilde{m}$ for temperatures different from the critical value $T_{c}$. As expected, one can see that for a temperature lower than $T_{c}$ (Fig. 2a), the maximum value of $P^{*}$ increases when the lattice size $L$ increases, while for a temperature greater than $T_{c}$, an increase of $L$ leads to a decrease of the corresponding peaks of $P^{*}$ (see Fig. 2b).

In other words, suppose we have a distribution function $P^{*}(\tilde{m})$ for a given $L$ (say for example, $L=16$ ) at a fixed temperature $T_{L=16}$. If $T_{L=16}<T_{c}$, a similar distribution will be obtained for a bigger lattice (e.g., $L=64$ ) at a different temperature $T_{L=64}$ such that $T_{L=16}<T_{L=64}<T_{c}$. Analogously, if $T_{L=16}>T_{c}$, we
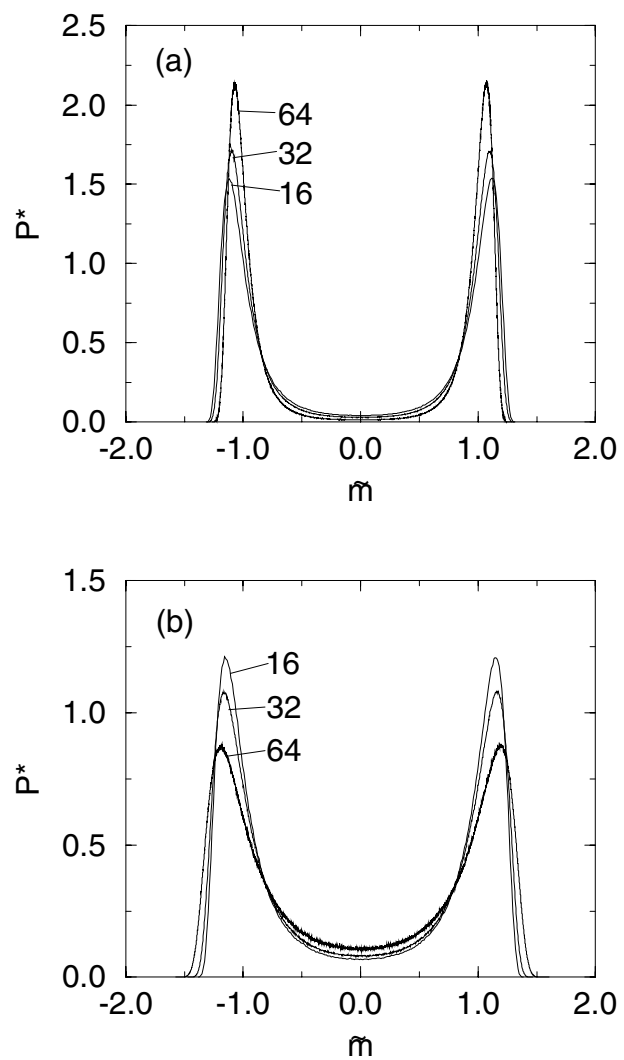

Figure 2. Scaling function $P^{*}(\tilde{m})$ for the spin-1/2 Ising model with $L=16,32$, and 64 at a fixed temperature $T$ : (a) lower than $T_{c}(T=2.2472)$ and (b) greater than $T_{c}(T=2.2831)$. The error bars have been ommited for clarity.

will have $T_{L=16}>T_{L=64}>T_{c}$. This fact suggests a mechanism to obtain the critical temperature, as well as the exponent $\nu$ and the universal distribution, for the system under study. Table I shows the temperatures of several lattice sizes we have used to obtain the distributions displayed in Fig. 3. These temperatures were evaluated as follows. For $L=64$ and a given temperature, for instance $T=2.2989$ in Table I, we compute the corresponding probability distribution of the order parameter, which will be the "reference" distribution. For other values of $L$, we search for the temperature $T_{L}$ which gives a distribution equivalent to the reference one. In this way, we obtain the data shown in the second column of Table I. Taking a different reference distribution, obtained at a different temperature for $L=64$, we have another set of $T_{L}$, and so on. All the distributions so obtained are depicted in Fig. 3. It means that each curve in Fig. 3 is in fact a superposition of six different distributions taking at the temperatures given in Table I.

Since one expects that the difference $\left|T_{L}-T_{c}\right|$ scales as $L^{-1 / \nu}$, where $\nu$ is the correlation length critical exponent, a finite-size scaling analysis can be done to estimate the critical values of the infinite system. In Fig. 4a, we have a plot of $T_{L}$ vs. $L^{-1 / \nu}$, with $\nu=1$, using the values of the first five columns of Table I, which confirms the exact exponent $\nu=1$ and gives $T_{c}=2.267(2)$. Another choice for this model is, of course, the corresponding critical temperature. 
TABLE I. Temperature for different lattice sizes at which the distribution $P^{*}(\tilde{m})$ for $L=16-48$ is the same as that obtained for $L=64$ at the shown temperatures (spin-1/2). Error in parentheses affects the last digits. The second and third columns correspond to temperatures greater than the critical one, and the two following columns correspond to temperatures lower than the critical one. The last column represents the data when $P^{*}(\tilde{m})$ for $L=64$ is obtained at $T_{c}$.

\begin{tabular}{cccccc}
\hline \hline Size & \multicolumn{5}{c}{ Temperature (in units of $J / k_{B}$ ) } \\
\hline 16 & $2.3923(11)$ & $2.3272(8)$ & $2.2477(8)$ & $2.1901(10)$ & $2.27221(52)$ \\
20 & $2.3666(8)$ & $2.3154(8)$ & $2.2502(8)$ & $2.2036(10)$ & $2.27092(52)$ \\
24 & $2.3502(8)$ & $2.3073(5)$ & $2.2528(5)$ & $2.2134(7)$ & $2.27015(52)$ \\
32 & $2.3288(5)$ & $2.2973(5)$ & $2.2563(5)$ & $2.2262(5)$ & $2.26963(26)$ \\
48 & $2.3089(5)$ & $2.2878(5)$ & $2.2604(5)$ & $2.2399(5)$ & $2.26937(26)$ \\
64 & 2.2989 & 2.2831 & 2.2624 & 2.2472 & 2.269184 \\
\hline \hline
\end{tabular}

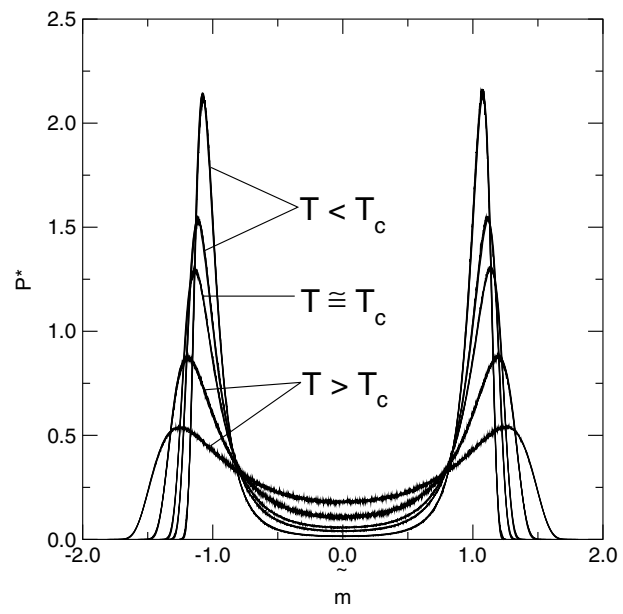

Figure 3. Normalized distribution $P^{*}(\tilde{m})$ for systems with lattice sizes and temperatures shown in Table I. Each curve is a supperposition of six different distributions taking with the data from this table.

In this case (very close to $T_{c}$, last column of Table I), however, it is known that $\left|T_{L}-T_{c}\right|$ scales as $L^{-(1+\theta) / \nu}$, where $\theta$ is the correction to scaling exponent [1]. In Fig. 4b, we plot the estimates $T_{L}$ as a function of $L^{-(1+\theta) / \nu}$ with $\nu=1$ and $\theta=2$ [14]. Linear regression gives $T_{c}=$ $2.2693(1)$ for the infinite system, which is in fact quite close to the exact one.

In order to measure the applicability of the present mechanism for obtaining the transition temperature from non-universal distributions, we also study the spin-1 Ising model. To have an idea of the value of $T_{c}$, one just performs short simulations in a range of temperatures to check whether the probability distribution $P(m)$ has single or double peak. Then, one proceeds according to the same manner already discussed for the spin-1/2 case. We fix the temperature and verify how the peaks of the distribution change if the lattice size $L$ increases. Fig. 5 shows the distributions obtained for lattice sizes $L=12,16,24$, and 32 at two different temperatures: $T=1.660$ and $T=1.720$. In the former case an increasing lattice size leads to increasing peaks, while in the latter case the height of the peaks decreases when the lattice becomes larger. Thus, we conclude that the transition temperature is between 1.660 and 1.720 , and hence we perform longer simulations in this temperature range.
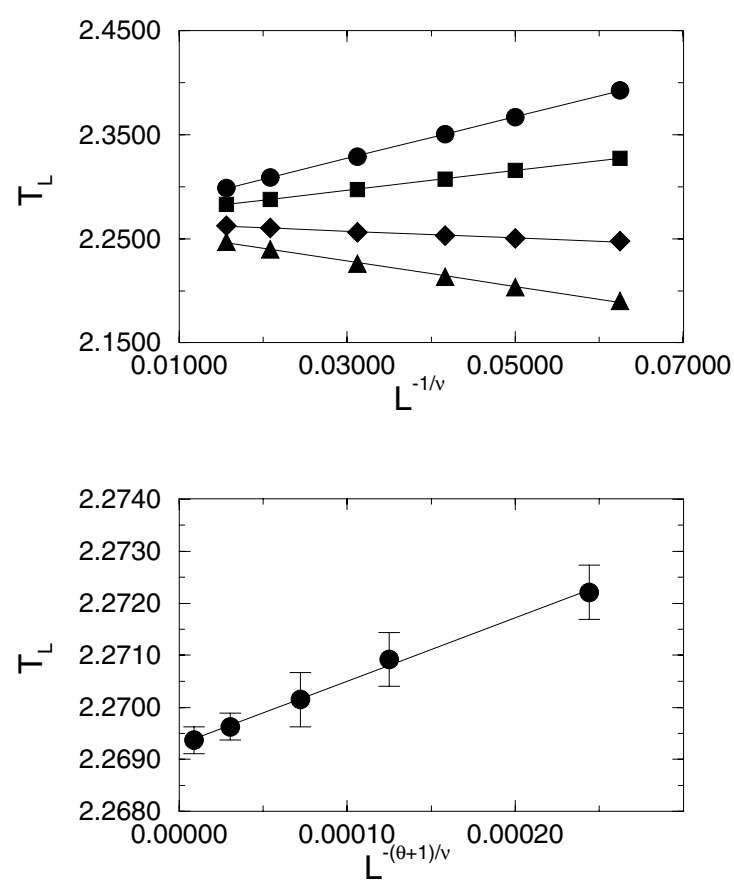

Figure 4. (a) Temperature $T_{L}$ as a function of $L^{-1 / \nu}$ with $\nu=1$. Different symbols correspond to different choices of the reference distribution (from top to bottom we have the results from the second to fifth columns of Table I). Error bars are smaller than the symbol sizes. (b) Temperature $T_{L}$ as a function of $L^{-(1+\theta) / \nu}$ with $\nu=1$ and $\theta=2$ taking the data of the last column of Table I.

The procedure now is the same as that we have done for the spin-1/2 model. We fix the temperature and compute $P^{*}(\tilde{m})$ for the lattice with $L=32$ (reference distribution). For a different $L$, we search for the temperature that gives a distribution equal to the reference one. Table II shows the temperatures so obtained. For each set of temperatures (which corresponds to each column of Table II), we plot $T_{L}$ vs. $L^{-1 / \nu}$ and vary the exponent $\nu$ until we get a straight line. Thus, each column of Table II gives an independent estimate of $\nu$ and also of the critical temperature $T_{c}$. Fig. 6 illustrates this procedure. By taking the mean value of these quantities, one obtains $\nu=1.0(1)$ and $T_{c}=1.6933(16)$, where the latter agrees well with the value $T_{c}=1.6935(10)$ [10]. After we have evaluated $T_{c}$, we ran a longer simulation on a larger lattice to determine, by this way, the universal 
distribution $P^{*}(\tilde{m})$. Fig. 7 shows the distribution $P^{*}(\tilde{m})$ on a $L=64$ lattice for spin-1/2 and spin-1 models, and confirms the fact that both systems belong to the same universality class, as already expected.
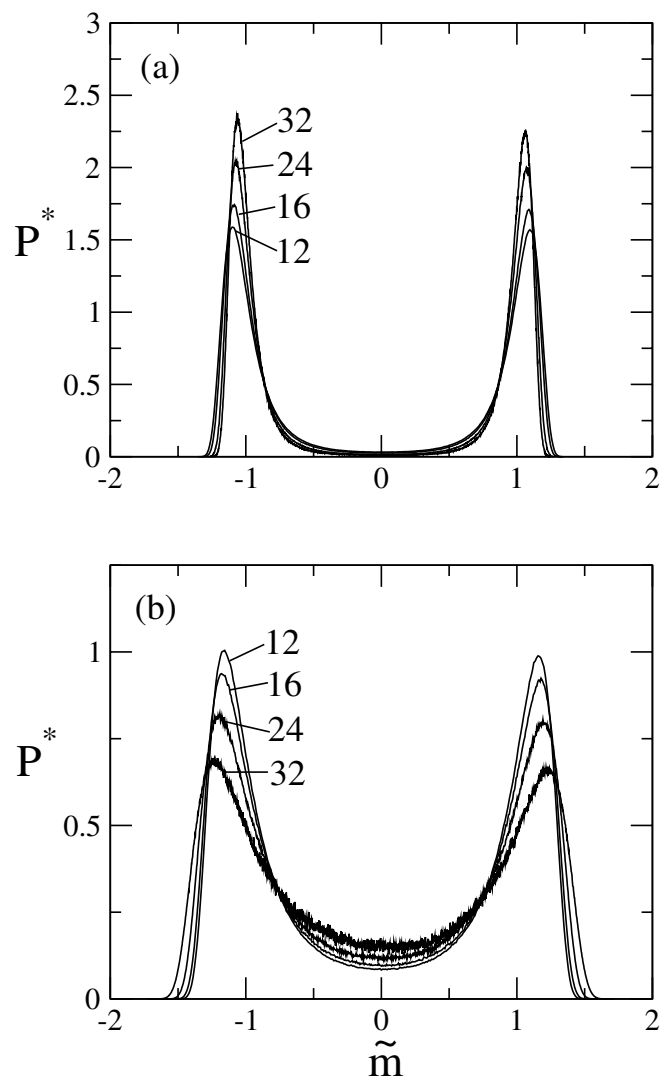

Figure 5. Scaling function $P^{*}(\tilde{m})$ for the spin-1 model on square lattices with $L=12,16,24$, and 32 at a fixed temperature $T$ : (a) lower than $T_{c}(T=1.660)$ and (b) greater than $T_{c}(T=1.720)$.

TABLE II. Temperature for different lattice sizes at which the distribution $P^{*}(\tilde{m})$ for $L=12-24$ is the same as that obtained for $L=32$ at the shown temperatures (spin-1). Error in parentheses affects the last digits. The second and third columns correspond to temperatures greater than the critical one, and the two following columns correspond to temperatures lower than the critical one.

\begin{tabular}{cllll}
\hline \hline Size & \multicolumn{5}{c}{ Temperature (in units of $J / k_{B}$ ) } \\
\hline 12 & $1.598(4)$ & $1.652(2)$ & $1.708(2)$ & $1.765(1)$ \\
16 & $1.624(2)$ & $1.664(2)$ & $1.705(2)$ & $1.747(1)$ \\
24 & $1.649(2)$ & $1.675(1)$ & $1.702(1)$ & $1.730(1)$ \\
32 & $1.660(2)$ & $1.680(1)$ & $1.700(1)$ & $1.720(1)$ \\
\hline \hline
\end{tabular}

\section{Conclusions}

The present approach, using just the order parameter distribution, seems to be a robust way to obtain the criticality of magnetic systems, regarding its universal and non-universal aspects. There is a clear distinction between the finite-size

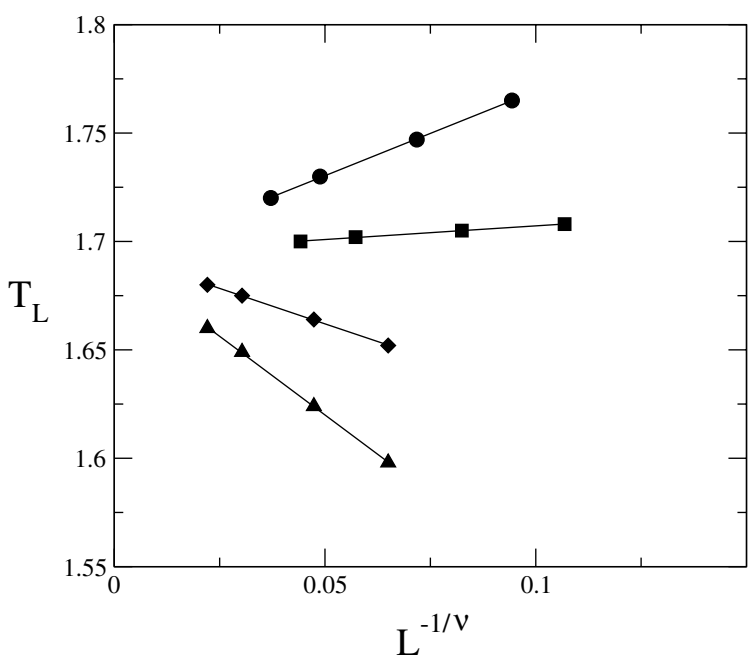

Figure 6. Temperature $T_{L}$ as a function of $L^{-1 / \nu}$ (spin-1). Different symbols correspond to different choices of the reference distribution (see Table II). The values of $\nu$ that give the best linear fit were (from top to bottom): $\nu=0.9(1), \nu=0.9(1), \nu=1.1(1)$, and $\nu=1.1(1)$. Error bars are smaller than the symbol sizes.

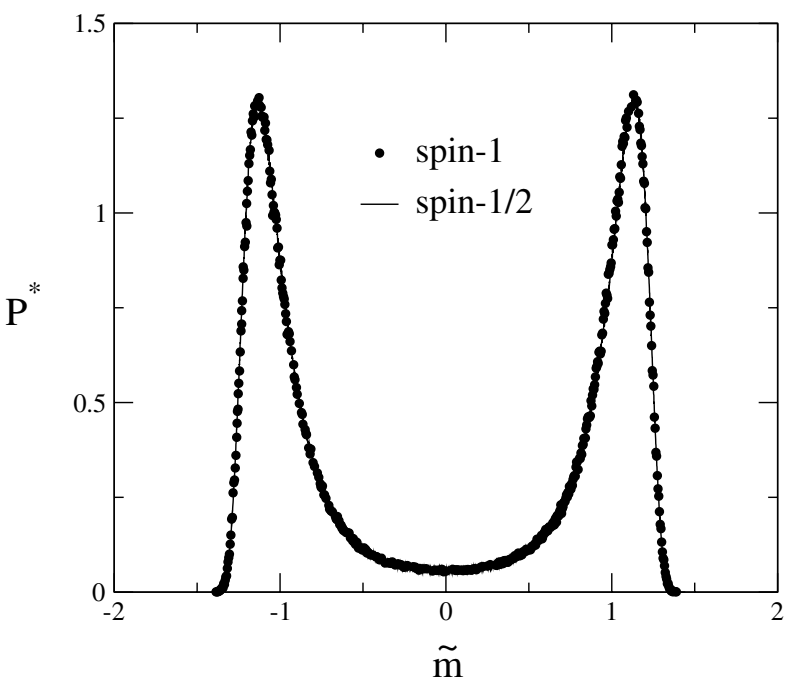

Figure 7. Universal function $P^{*}(\tilde{m})$ for lattice size $L=64$ at temperatures obtained in this work: $T=2.2693$ for spin- $1 / 2$ and $T=1.6933$ for spin- 1 .

behavior of $P^{*}$ close to the critical temperature (scaling with $L^{-(1+\theta) / \nu}$ ) or away from it (scaling with $L^{-1 / \nu}$ ), as depicted in Figs. 4 and 6. It also seems, at first sight, that there is a limitation regarding the size of the lattices which could be considered. For instance, we have used here lattice sizes which are smaller than that from the reference distribution. Nevertheless, this limitation is not so drastic since in the spin-1 model we considered the reference distribution for $L=32$ and with lattices smaller than this value the results prove quite accurate. One can, of course, consider lattices larger than that of the reference distribution. 
We feel, however, that reweighting the distribution is more easily done for smaller systems. Application of the present procedure to other models (pure and random), as well as to multicritical behavior, will be very welcome; some are now in progress.

\section{Acknowledgments}

We would like to thank R. Dickman for fruitful discussions and a critical reading of the manuscript. Financial support from the Brazilian agencies CNPq, CAPES, FAPEMIG and CIAM-02 49.0101/03-8 (CNPq) are gratefully acknowledged.

\section{References}

[1] K. Binder, Z. Phys. B 43, 119 (1981).

[2] A.D. Bruce, J. Phys. C 14, 3667 (1981).

[3] D. Nicolaides and A.D. Bruce, J. Phys. A 21, 233 (1988).

[4] J.A. Plascak and D.P. Landau, Phys. Rev. E 67015103 (R) (2003).

[5] M.M. Tsypin and H.W.J. Blöte, Phys. Rev. E 62, 73 (2000).
[6] A. D. Bruce and N. B. Wilding, Phys. Rev. Lett. 68, 193 (1992); N. B. Wilding and A. D. Bruce, J. Phys.: Condens. Matter 4, 3087 (1992); N. B. Wilding, Phys. Rev. E 52, 602 (1995).

[7] K. Rummukainen, M. Tsypin, K. Kajantie, M. Laine, and M. Shaposhnikov, Nucl. Phys. B 532, 283 (1998).

[8] C. Alexandrou, A. Borici, A. Feo, P. de Forcrand, A. Galli, F. Jegerlehner, and T. Takaishi, Phys. Rev. D 60, 034504 (1999).

[9] M.E. Fisher in Critical Phenomena, edited by M.S. Green (Academic, New York, 1971).

[10] J. Adler and I.G. Enting, J. Phys. A 17, L275 (1984).

[11] J.A. Plascak, A.M. Ferrenberg, and D. P. Landau, Phys. Rev. E 65, 066702 (2002)

[12] R. Dickman and W.C. Schieve, J. Physique 45, 1727 (1984).

[13] A.M. Ferrenberg and R.H. Swendsen, Phys. Rev. Lett. 61, 2635 (1988).

[14] P. Calabrese, M. Caselle, A. Celi, A. Pelisseto, and E. Vicari, J. Phys. A 33, 8155 (2000); J. Salas and A. Sokal, J. Stat. Phys. A 98, 551 (2000); S.L.A. de Queiroz, J. Phys. A 33, 721 (2000). 